\title{
Current-Controlled Nanospray Ionization Mass Spectrometry
}

\author{
Alexei Gapeev, Alberto Berton, and Daniele Fabris \\ Department of Chemistry and Biochemistry, University of Maryland, Baltimore County, Baltimore, \\ Maryland, USA
}

\begin{abstract}
The hypothesis that direct determination of electrospray current would provide a viable method for maintaining spray stability to enable optimal nanospray analysis was tested by building a feedback apparatus capable of reading the current and readjusting the emitter voltage in real time. The apparatus consists of a current-sensing circuit that reads the voltage drop across a resistor located between the high-voltage power supply and the nanospray emitter. A low voltage proportional to the observed current is generated and sent to a data acquisition card. The information is used by a proportional-derivative-integral (PID) algorithm to calculate the magnitude of a low-voltage signal that is used to control the power supply output. Any variation of current across the sensing resistor is thus counteracted by an opposite-direction variation of the high voltage applied to the nanospray emitter. In this way, the apparatus adjusts the emitter voltage to achieve a preset value of current, which it strives to maintain over time in spite of any possible variation of the parameters influencing the spray regime. Preliminary results have shown that the feedback apparatus is capable of establishing and maintaining stable spray for samples that are usually considered challenging in traditional voltage-controlled analysis, such as those consisting of nucleic acid solutions with high salt loads. For these types of samples, the total ion count recorded in current-controlled mode was significantly more stable than that observed in voltage-controlled mode. At the same time, overall signal intensities and signal-to-noise ratios were also significantly improved. Setting the target nanospray current to a predefined value and letting the apparatus reach the target without operator intervention enabled the acquisition of viable data from solutions containing up to $2.5 \mathrm{M}$ ammonium acetate, which are ordinarily difficult by traditional manual tuning. A deeper understanding of the current-voltage relationships for samples of very different compositions is expected to enable one not only to predict the target current that should be used for a certain analysis, but also to devise algorithms to change such target as a function of predictable variations of sample properties and analytical conditions. This will allow for optimal performance to be maintained during on-line gradient chromatography in which the nature of the sprayed solution may vary very widely during the course of the analysis. (J Am Soc Mass Spectrom 2009, 20, 1334-1341) (C) 2009 Published by Elsevier Inc. on behalf of American Society for Mass Spectrometry
\end{abstract}

$\mathrm{T}$ The development of low-flow electrospray interfaces has greatly benefited the mass spectrometric analysis of samples of limited availability, attributed to intrinsically high ion yields and modest analyte consumption [1-3]. Typical implementations use emitters obtained by pulling capillaries made of fused silica or borosilicate glass to produce a finely tapered tip [4]. A constant voltage is supplied through a thin metal layer deposited onto the needle surface [1, 4], a liquid junction [2, 3], or a metal wire inserted from the capillary back end to make contact with the sample solution $[5,6]$. The electric field between emitter tip and counter-electrode induces electrostatic dispersion of the solution into charged droplets that are readily entrained into the atmosphere-vacuum interface of the electros-

Address reprint requests to Dr. Daniele Fabris, University of Maryland, Baltimore County, Department of Chemistry and Biochemistry, 1000 Hilltop Circle, Baltimore, MD 212250. E-mail: fabris@umbc7.umbc.edu pray source. Flow rates in the nanoliter per minute range are maintained by the capillary action established by the dispersion process at the emitter tip, with no need for positive pressure exerted by solvent pumps. The process produces droplets that are significantly smaller [7] than those obtained from conventional electrospray operating at higher flow rates $[8,9]$. Because of the more efficient solvent evaporation afforded by the smaller size, such droplets can be generated closer to the interface inlet without sheath gas. As a result, a larger proportion of the initial analyte enters the mass spectrometer rather than being released into the atmosphere or deposited onto the inlet surface, thus providing increased analytical sensitivity [4].

In any mass spectrometric analysis, signal stability is a critical figure of merit determining spectral quality and analytical performance. Ideally, any ion source should be capable of sustaining maximal ion production throughout the detection duty cycle. In electros-
(C) 2009 Published by Elsevier Inc. on behalf of American Society for Mass Spectrometry. $1044-0305 / 09 / \$ 32.00$

doi:10.1016/j.jasms.2009.03.007
Published online March 14, 2009 Received February 9, 2009 Revised March 6, 2009 Accepted March 9, 2009 
pray ionization, signal stability is directly correlated to the spray stability achieved during the experiment, which in turn is influenced by the interplay of factors including applied voltage, flow rate, solution composition, and emitter geometry [10-15]. Valuable insights into the relationship between signal stability and analytical performance have been obtained by investigating the different spray modes identified over the years [16-20], which have led to practical guidelines for successful analysis. In general, most of the experimental conditions are predetermined by the specific hardware design, type of analysis, and solution composition. Others, including emitter position and applied voltage, can be optimized immediately before analysis and are expected to remain constant throughout the experiment. Although most experiments involve only minor tuning to achieve stable spray, the procedure may become rather challenging for samples containing elevated concentrations of salts and detergents. The ever possible occurrence of corona discharge can hamper spray stability when the analysis is performed in negative-ion mode [21]. Further, maintaining a stable spray may become problematic when the solution composition varies over the course of the analysis—such as during on-line gradient chromatography-which may result in progressive drift of the optimal voltage.

Fast imaging techniques have been used to match the various spray modes with characteristic regions of a typical current-voltage diagram [18, 22-25]. In the case of analyte solutions containing organic solvents, such as methanol and acetonitrile, a strong correlation has been observed between cone-jet sprays and stable signal profiles associated with desirable spectral performance. This observation provided the rationale for feedback systems capable of monitoring the actual shape of the spray plume and immediately readjusting the applied voltage or emitter position to maintain optimal signal [26]. This type of approach, however, does not account for the fact that less-than-ideal samples may not always allow for a stable cone-jet shape to be obtained within the range of sampled conditions. Determining the actual electrospray current constitutes a more direct means for monitoring the spray/signal stability [27-30], which could afford greater flexibility for dealing with problematic situations in which the desired plume morphology cannot be obtained. We have tested this hypothesis by building a feedback system that adjusts the emitter voltage according to real-time readings of electrospray current. In this report, we describe its design and provide initial characterization using samples and analytical conditions, which are usually considered as challenging in traditional voltage-controlled analysis.

\section{Experimental}

The spray region of a typical electrospray source can be described by a simple electrical diagram in which sprayed droplets provide the connection between the emitter tip and the source orifice [31], thus completing the electrical circuit powered by the high-voltage power supply (HV; Scheme 1).

According to Kirchhoff's first law, the current carried by the charged droplets $\left(i_{E S I}\right)$ could be measured at any point of the circuit. However, considering that a fraction of the charged particles is discharged at the counter-electrode $\left(i_{c e}\right)$, whereas the remainder enters the orifice and is eventually neutralized in other parts of the mass spectrometer $\left(i_{m s}\right)$, points located between emitter and power supply are uniquely suited for providing an overall reading of the current flowing through the circuit. A classical method for performing current determinations involves measuring the voltage drop across a sensing resistor $\left(R_{S}\right)$ of known value. Considering that typical currents afforded by low-flow electrospray rarely exceed microampere levels and that applied voltages are usually in the $1-$ to $2-\mathrm{kV}$ range, we calculated that a $100-\mathrm{k} \Omega$ resistor would allow for an upper limit exceeding by at least one order of magnitude the maximum expected current. Placed in series between power supply and electrospray emitter, the sensing resistor introduces a voltage drop proportional to the current flow, which can be measured by a voltmeter or voltage comparator (V). In the initial prototype, a Meterman 38XR digital multimeter (Everett, WA, USA) was used to generate an opto-isolated serial (RS-232) output stream representative of the measured drop. In an improved implementation, an optoisolated sensor was used to provide a ground-referenced voltage proportional to the recorded spray current (Scheme 2).

The circuit uses an optocoupler photodiode $\left(\mathrm{U}_{3}\right)$ to isolate the circuitry held at the high-voltage sustaining typical spray operation (A section) from the low-voltage circuitry that provides the output proportional to the electrospray current (B section). For this reason, the

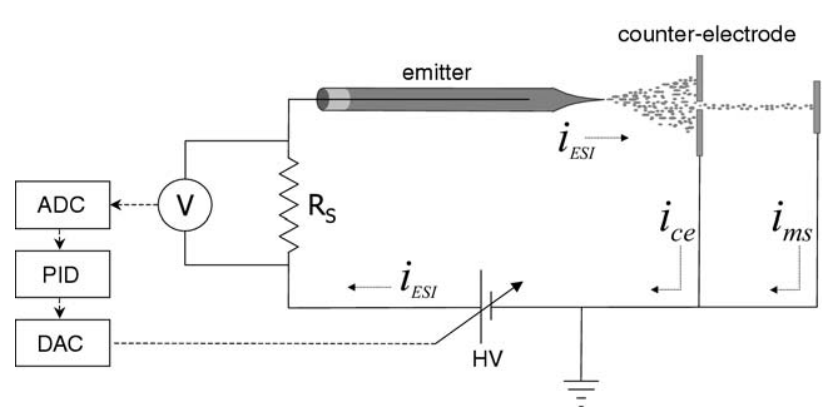

Scheme 1. Representation of the spray region as an electric circuit. HV is the high-voltage supply powering the circuit; $i_{E S I}$ indicates the overall current carried across the gap between emitter and counter-electrode; $i_{c e}$ is the fraction of current discharged at the electrode; $i_{m s}$ is the fraction discharged elsewhere in the mass spectrometer; $\mathrm{R}_{\mathrm{S}}$ is the current sensing resistor; $\mathrm{V}$ is a voltage-measuring device (see Scheme 2); ADC and DAC represent the analog-to-digital and digital-to-analog components of the data acquisition card; PID is the proportional-derivative-integral algorithm that calculates output voltage values based on input signal from the current sensing circuit (see Experimental section). 


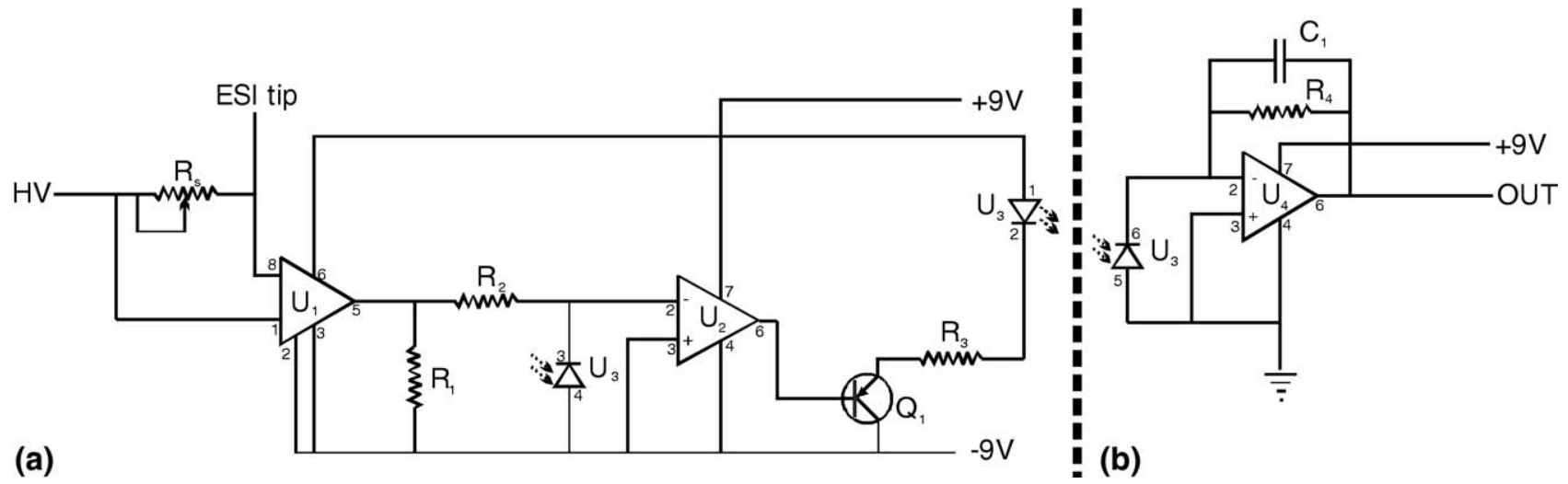

Scheme 2. Electronic schematic of the current sensing circuit, with (a) and (b) representing the highand low-voltage regions, respectively (see Experimental section). The different electronic components include: AD626ANZ as $\mathrm{U}_{1} ; \mathrm{TLV} 2371 \mathrm{IP}$ as $\mathrm{U}_{2}$ and $\mathrm{U}_{4} ; \mathrm{HCNR} 200-000 \mathrm{E}$ as $\mathrm{U}_{3} ; 2 \mathrm{~N} 3906$ as $\mathrm{Q}_{1} ; \mathrm{R}_{\mathrm{S}} 100 \mathrm{k} \Omega$; $\mathrm{R}_{1} 4.6 \mathrm{k} \Omega ; \mathrm{R}_{2} 330 \mathrm{k} \Omega ; \mathrm{R}_{3} 550 \Omega ; \mathrm{R}_{4} 680 \mathrm{k} \Omega ; \mathrm{C}_{1} 10 \mathrm{pF}$.

former is floated at the electrospray voltage, whereas the latter is grounded. Each section is powered by a separate $9-\mathrm{V}$ battery. The floating circuit detects the voltage drop across $R_{S}$, which is amplified by the differential amplifier $U_{1}$, whereas the function of $U_{2}$ is to further amplify the voltage and to linearize the optocoupler response. In the grounded circuit, $\mathrm{U}_{4}$ amplifies the voltage generated by the optocoupler and provides a DC voltage in the $0.5-$ to $8.5-\mathrm{V}$ range, which is representative of the nanospray current.

The output from the current-sensing circuit was processed by a PC laptop equipped with a data acquisition card (DAQCard-6024E, National Instruments, Austin, TX, USA). The analog-to-digital converter of the card (ADC, Scheme 1) translates the analog signal from the sensing circuit into input information for a proportionalderivative-integral (PID) algorithm written in LabVIEW (National Instruments, Austin, TX, USA). According to the algorithm, the actual current reading $(C R)$ is compared to the desired set-point $(S P)$ to calculate the current error $(e)$ at any given time $(t)$ :

$$
e=S P-C R
$$

This value is then used to calculate the output signal $u(t)$ as the sum of three corrective terms corresponding to the proportional $(\mathrm{P})$, integral (I), and derivative (D) terms of the PID algorithm:

$$
u(t)=K_{c}\left[e+\frac{1}{T_{i}} \int_{0}^{t} e d t+T_{d} \frac{d e}{d t}\right]
$$

in which $K_{c}$ represents the gain constant, whereas $T_{i}$ and $T_{d}$ correspond to the integral and derivative time constants, respectively [32]. The first corrective term contributes a change that is proportional to the magnitude of the current error; the integral term introduces a correction proportional to both the magnitude and duration of the error in the interval considered; the derivative term accounts for the rate of error change. The new value calculated by the algorithm is translated by the digital-to-analog converter of the card (DAC, Scheme 1) into a signal in the $\pm 10-\mathrm{V}$ range, which is used to control a Tennelec (Oak Ridge, TN, USA) TC95 high-voltage power supply (HV) that provides the actual emitter voltage. The various tuning parameters necessary to control the apparatus operations were made accessible to the operator through a graphic-user interface (GUI) written in LabVIEW.

The current control apparatus was tested by analyzing nucleic acid standards obtained from IDT (Coralville, IA, USA) and used without further purification. Actual samples were prepared by adding the desired volume of analyte stock to ammonium acetate solutions of different concentrations, as indicated in the text. In a typical experiment, a $5-\mu \mathrm{L}$ aliquot of analyte solution with indicated concentration was loaded into a borosilicate glass needle positioned around $1 \mathrm{~mm}$ in front of the capillary inlet of the mass spectrometer. The emitters were produced in house by using a P-87 (Sutter Instruments, Novato, CA, USA) micropipette puller. An initial ionization voltage of about $800 \mathrm{~V}$ was typically applied through a $\mathrm{Pt}$ wire inserted from the back end of the emitter, but the actual voltage was subsequently allowed to vary between user-defined boundaries under feedback control. All analyses were performed on a Bruker Daltonics (Billerica, MA, USA) Apex III Fourier transform ion cyclotron resonance (FT-ICR) [33] mass spectrometer equipped with a 7 Tesla actively shielded superconducting magnet and an Apollo electrospray source that has been modified in-house to enable lowflow operations and to include a heated metal capillary. Before mass analysis, ions were typically accumulated for $1 \mathrm{~s}$ in a radiofrequency (rf)-only hexapole element located immediately after the high-pressure region of the electrospray source [34,35]. Spectra were acquired in negative-ionization mode and processed by ApexControl 2.0 (Bruker Daltonics). Spectra were externally cali- 
brated using a $1 \mathrm{mg} / \mathrm{mL}$ solution of CsI, which produces a series of peaks throughout the mass range of $500-7000 \mathrm{~m} / \mathrm{z}$.

\section{Results and Discussion}

The ability of the feedback controller to respond in real time to the electrospray current was tested by recording actual current and corresponding output voltage as a function of time. A $10 \mu \mathrm{M}$ solution of a 17 mer deoxyoligonucleotide (Top17) in $10 \mathrm{mM}$ ammonium acetate displayed typical patterns characterized by wide current oscillations at the onset, followed by gradual dampening and stabilization around a preset target of $75 \mathrm{nA}$ (Figure 1a). At the same time, the emitter voltages applied by the controller were relatively large at the beginning, but decreased gradually toward a stable level, in this case around $-930 \mathrm{~V}$ (Figure 1b). The corresponding mass spectra acquired within the flat region of the diagram afforded the desirable spectral features that are typically associated with sprays operating in a cone-jet regime (see following text). The wide oscillations observed at the beginning were consistent with the cyclic nature of the feedback operations, which involves sending voltages that are alternatively higher or lower than the previous value to approach, and to eventually reach the correct voltage associated with the target current. In this case, the successive approximations resulted in progressively smaller oscillations

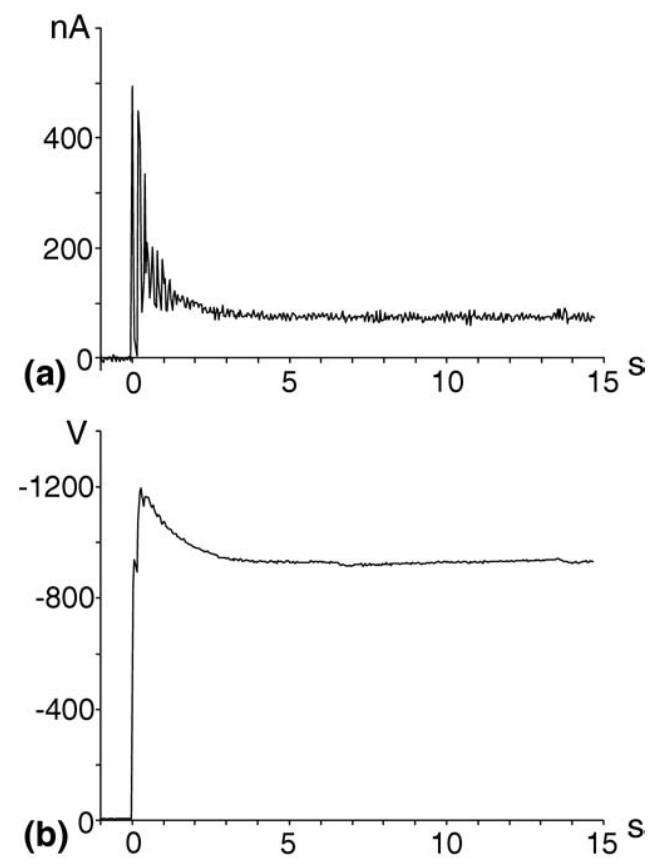

Figure 1. Current reading (a) and emitter voltage (b) recorded over time under feedback control. The sample consisted of a 10 $\mu \mathrm{M}$ solution of a 17 mer deoxyoligonucleotide (Top17) in $10 \mathrm{mM}$ ammonium acetate. Time 0 marks the point at which the current controller was turned on with a current set-point of $75 \mathrm{nA}$ and a maximum allowable voltage of $1300 \mathrm{~V}$ (see Results and Discussion section). around the desired value, which was reached after about $3 \mathrm{~s}$ of feedback operation under the selected conditions. The amplitude variability recorded in the diagram's flat region amounted to a $\pm 5.3 \mathrm{nA}$ standard deviation from the mean, which was not significantly greater than the baseline noise of $\pm 3.9 \mathrm{nA}$ determined in the absence of feedback activity (i.e., in the region preceding time 0 in Figure 1a).

Feedback cycles were completed with a $25-\mathrm{Hz}$ frequency to allow for the spray to closely track each voltage change, while minimizing possible effects induced by the hysteresis intrinsic in spray processes [28, 36, 37]. This setting was adjusted to prevent the PID algorithm from calculating an output before the effects of the previous approximation could be felt, which could result in excessive increases of the applied voltage. Although the current sensing circuit could be readily operated at up to $200 \mathrm{kHz}$, the selected iteration rate was sufficiently slow to integrate current oscillations induced by high-frequency pulsation phenomena that have been observed in the kilohertz range at the flow rates typical of regular electrospray operations (e.g., $\mu \mathrm{L} / \mathrm{min}$ ) [18]. At the same time, it was sufficiently fast to enable the sampling of low-frequency instabilities expected to fall in the $2-$ to $10-\mathrm{Hz}$ range at the low flow rates obtained in the absence of a syringe pump (e.g., $\mathrm{nL} / \mathrm{min}$ ) [18, 20]. The nearly 3-s delay necessary to achieve stability in the absence of established spray (Figure 1a) provides an upper boundary for the overall response time afforded by the apparatus. This interval accounts for the time necessary for the electric field to drive the solution toward the emitter tip, to pull the meniscus out, and to initiate dispersion of sample in the gap between emitter and counter-electrode, which results in current onset. In the presence of established spray, however, this response will be expected to be much more rapid, especially in the case of chromatographic applications in which the controller will be required to react to very gradual variations in the conditions.

Oligonucleotide solutions containing increasing amounts of ammonium acetate were also used to evaluate the spectral performance achieved in currentcontrolled mode. This choice was based on the rationale that high salt concentrations tend to have adverse effects on the analysis of nucleic acids in negative-ion mode, which include not only spray instability, but also possible signal suppression and formation of nonspecific adducts [38-40]. Figure 2 provides a comparison of total ion count (TIC) diagrams obtained from a $10 \mu \mathrm{M}$ solution of Top17 in $400 \mathrm{mM}$ aqueous ammonium acetate. The dashed line corresponds to the normalized TIC obtained in traditional voltage-controlled mode, according to which the voltage supplied to the emitter was optimized immediately before analysis and was left unchanged for the entire experiment. In this case, tuning optimization was accomplished by finding a suitable signal and by iteratively readjusting source parameters to obtain acceptable signal intensity and 


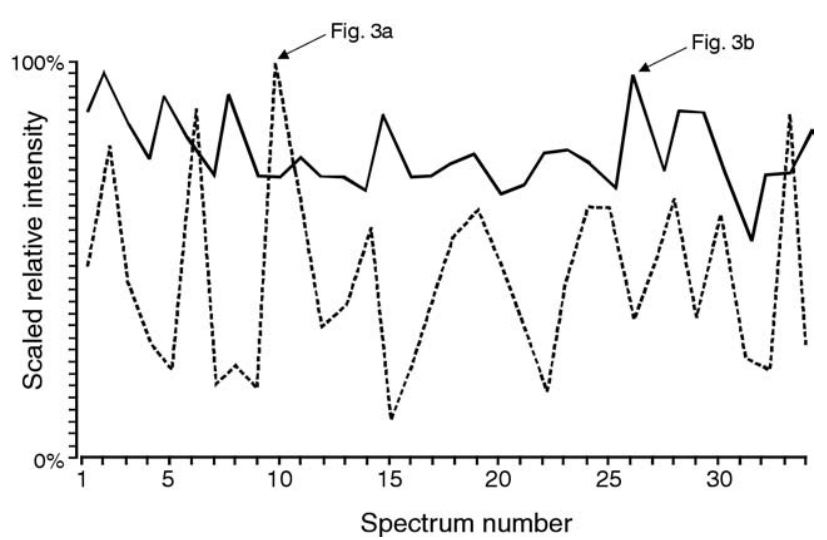

Figure 2. Total ion count (TIC) plots obtained by nanospray-FTICR analysis of a10 $\mu \mathrm{M}$ solution of Top17 in $400 \mathrm{mM}$ aqueous ammonium acetate without (dashed line) and with (solid) feedback control. The graphs were plotted on the same ordinate scale by setting the most intense spectrum as $100 \%$ and expressing the remaining points as relative intensity.

stability, as it would be normally done for ideal samples (e.g., organic co-solvent, low ionic strength, positive-ion mode). The solid line, instead, represents the normalized TIC provided by the same sample/emitter in current-controlled mode. In this case, the target current was set arbitrarily to $100 \mathrm{nA}$, whereas the supplied voltage was allowed to vary between -600 and -1400 $\mathrm{V}$ in response to the current readings. Although both diagrams reveal distinctive spectrum-to-spectrum fluctuations, the one obtained at constant voltage presents an overall $\pm 53 \%$ relative standard deviation (RSD), compared with the significantly better $\pm 12 \%$ RSD achieved under feedback control.
In comparing TIC data recorded by the mass analyzer with actual current readings from the sensing circuit, it is important to remember that only a fraction of all the charged particles dispersed in the gap is actually transmitted to the analyzer (Scheme 1), and only a fraction of these happens to fall in the mass range selected for detection. In addition, the instrumental setup used in this study included an rf-only hexapole for ion accumulation and collisional cooling before mass analysis [34, 35]. A typical 1-s accumulation interval can result in substantial signal averaging, thus abating the effects of any spray instabilities occurring on a shorter timescale. Nevertheless, the benefits of maintaining a stable current throughout the accumulation interval are evident not only from a higher average TIC through the entire experiment $\left(2.3 \times 10^{7}\right.$ versus $9.2 \times 10^{5}$ arbitrary counts), but also from the greater signal-to-noise ratios $(\mathrm{S} / \mathrm{N})$ afforded by individual mass spectra. For example, the spectra marked by arrows in Figure 2 afforded base peaks with an intensity of $4.0 \times 10^{5}$ arbitrary counts and close to $12 \mathrm{~S} / \mathrm{N}$ under traditional voltage control (Figure $3 a$ ) versus $1.6 \times 10^{8}$ arbitrary counts and roughly 10,000 S/N under current control (Figure $3 b$ ). The same observation was verified across the board for all spectra included in the TIC diagrams. The reproducibility of these results was tested by repeating analysis of the same sample in different days and with different emitters. In these experiments, an average $\mathrm{S} / \mathrm{N}$ of $12.0 \pm 1.4$ was obtained under voltage control, whereas an average $\mathrm{S} / \mathrm{N}$ of $8695 \pm 1676$ was observed after activating the feedback apparatus. The rather wide standard deviations afforded by these results were attributed to the fact that emitters were

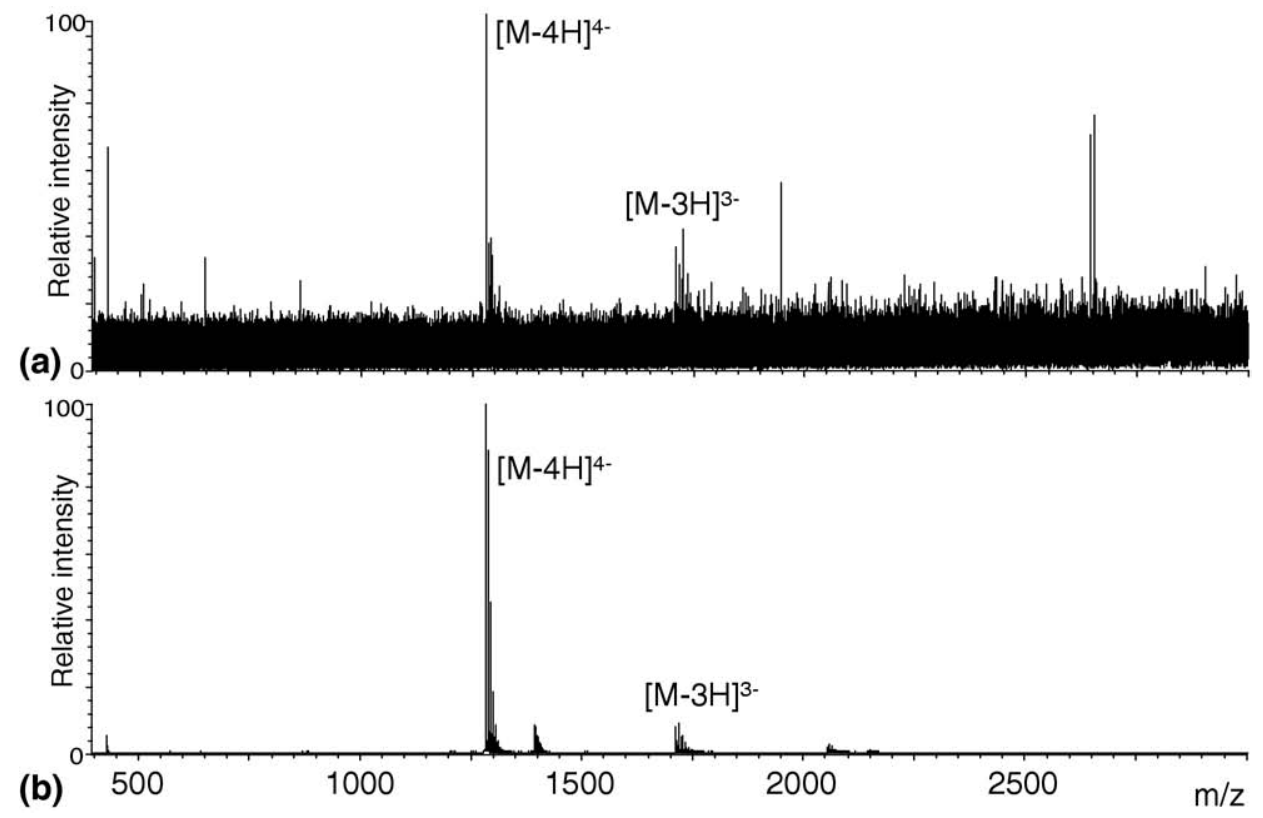

Figure 3. Nanospray-FT-ICR spectra obtained from a $10 \mu \mathrm{M}$ solution of Top17 in $400 \mathrm{mM}$ aqueous ammonium acetate without (a) and with (b) feedback control. These data correspond to the spectra marked on the TIC diagrams (Figure 2). 
pulled in house and used without actual quality control. Irrespective of the effects of variable emitter geometry, data obtained under current control were always significantly better than those obtained in the same experiment with normal tuning, thus highlighting the benefits of feedback operation.

In the present implementation, set-point current and allowable voltage range represent the critical parameters that require optimization for satisfactory operation. Proper settings are not likely to be known a priori because of the complex interplay of the different factors that may influence the spray's electrical properties and its current-voltage characteristics. Although approximate values could be gleaned from systematic studies of current-voltage plots obtained under a wide variety of experimental conditions [22, 23, 28, 36, 41-50], more focused experiments replicating the prospected analytical conditions will still be necessary for identifying optimal settings. An example is represented here by the survey of oligonucleotide solutions containing increasing concentrations of electrolyte, prompted by our continued interest in samples of relatively high ionic strength [40]. In this case, current-voltage profiles for 10 $\mu \mathrm{M}$ solutions of Top17 in 10, 50, 200, and $500 \mathrm{mM}$ ammonium acetate were obtained by spraying each sample in traditional voltage-controlled mode, which involved increasing stepwise the emitter voltage, and then recording the corresponding current after a brief stabilization period. The plots display only points that afforded sufficient signal stability to enable acquisition of actual mass spectrometric data (Figure 4). Therefore, each curve clearly identifies a viable voltage range delimited on one end by the minimum setting capable of producing current onset, on the other by the value inducing extreme spray instability and arcing. Despite the fact that these experiments relied on capillary action to maintain flow to the emitter tip, the results were consistent with those obtained in the presence of posi- tive pressure exerted by a syringe pump [31]. Also in this case, the curves displayed slopes increasing with salt concentration, as expected from the decrease of resistivity in the gap between emitter and counterelectrode associated with an increased supply of charge-carrying species. At the same time, the widening error bars highlighted the increasing instability at higher electrolyte concentrations (Figure 4), which usually afflicts the analysis of samples of high salt content. When values of current comprised within the viable ranges were used as preset targets for current-controlled operations, the feedback apparatus was always capable of meeting the respective voltages, while dampening unwanted oscillations.

These observations have important practical consequences on the procedure of spray optimization necessary for satisfactory analysis. In fact, under conditions characterized by wide current swings, such as those associated with high salt concentrations, it is often very difficult to find an initial signal that, although weak, would still allow the operator to begin tuning in traditional voltage-controlled mode. This was the case of a $10 \mu \mathrm{M}$ Top17 solution in $2.5 \mathrm{M}$ aqueous ammonium acetate, which failed to provide tunable signals despite repeated attempts to adjusting the applied voltage and emitter position. In contrast, a sufficiently stable current was readily achieved by turning on the feedback apparatus, which allowed for the acquisition of the data shown in Figure 5a. This spectrum was obtained by dialing a 200-nA target current and $1200 \mathrm{~V}$ upper voltage limit, which were comprised within the acceptable boundaries observed at somewhat lower concentrations of ammonium acetate (e.g., Figure 4). This example demonstrates how the feedback controller dispenses with having to identify a viable signal for optimization, but proceeds without operator intervention to search for the actual emitter voltage corresponding to the target current. The fact that the PID algorithm

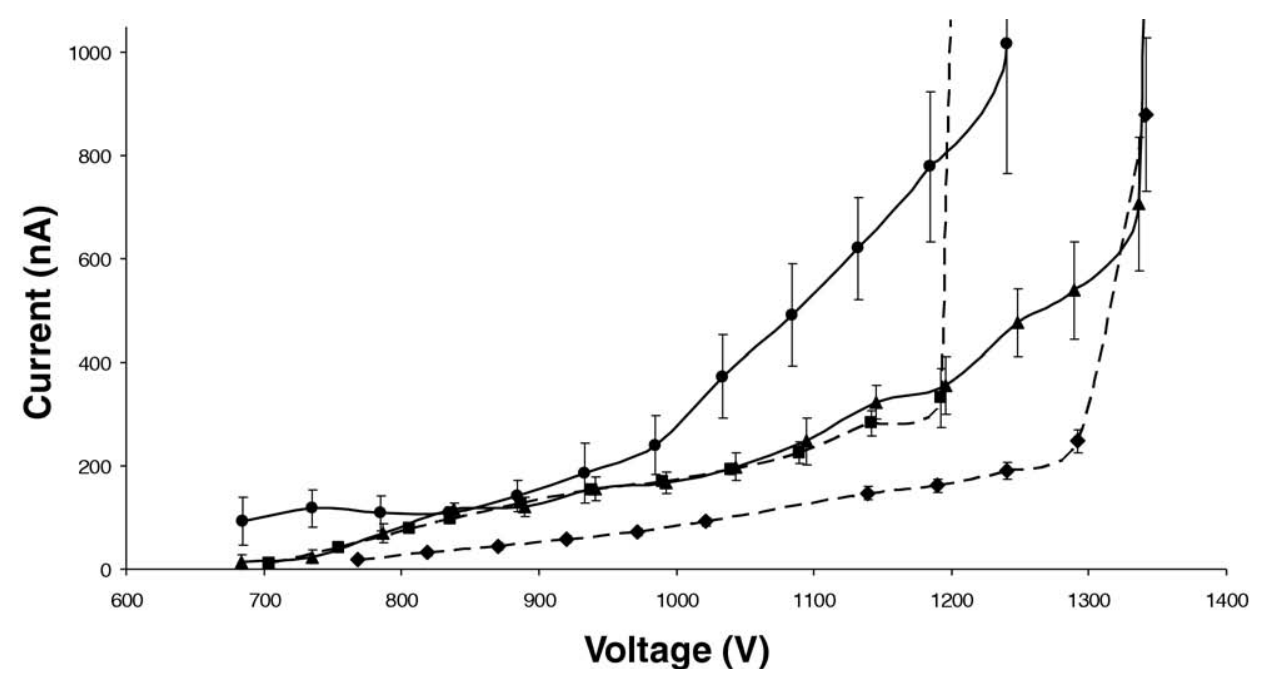

Figure 4. Current-voltage curves obtained without feedback control from $10 \mu \mathrm{M}$ solutions of Top17 in $10(\diamond), 50(\mathbf{\square}), 200(\boldsymbol{\Delta})$, and $500 \mathrm{mM}(\bullet)$ ammonium acetate. 


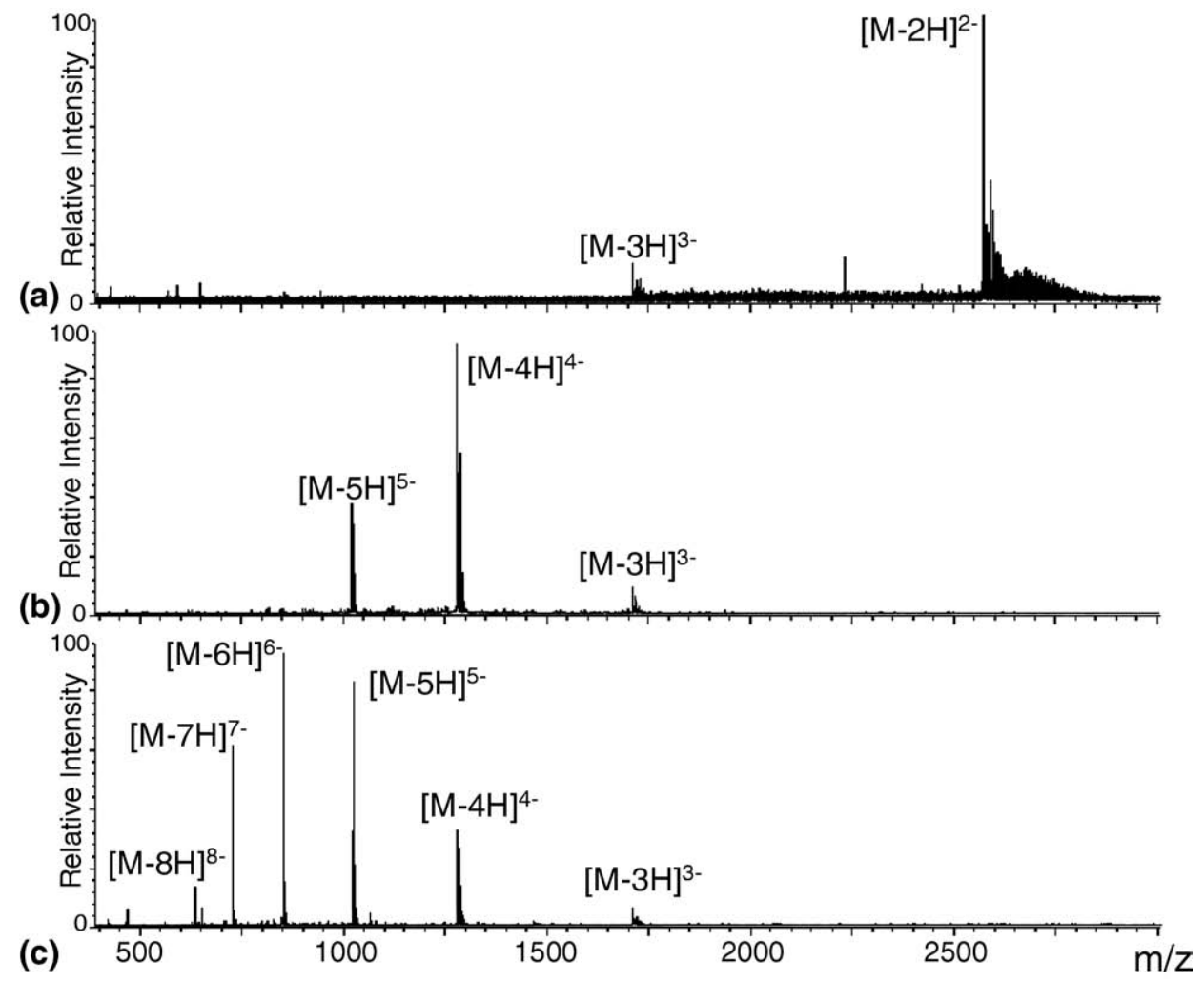

Figure 5. Nanospray-FT-ICR spectra obtained from $10 \mu \mathrm{M}$ Top17 in $2.5 \mathrm{M}$ aqueous ammonium acetate with feedback control (a), and in $10 \mathrm{mM}$ aqueous ammonium acetate without (b) and with (c) feedback control.

does not directly rely on the intensity of any preselected signal allows for this approach to be successful without prior knowledge of the content of the analyte solution and its effects on spray stability.

Finally, a close examination of the nucleic acid data obtained in the study revealed a tendency for the current-controlled mode to shift the observed charge state distributions toward higher charges, especially at the lower end of the salt concentrations tested thus far. This trend is clearly highlighted by comparing the spectra provided by a $10 \mu \mathrm{M}$ solution of Top 17 in 10 $\mathrm{mM}$ aqueous ammonium acetate, with and without feedback control (Figure $5 b$ and c). In fact, charge states ranging from -3 to -5 were detected after manual tuning in voltage-controlled mode, whereas a distribution ranging between -3 and -8 was readily observed after activating the current control apparatus with a set spray current of $100 \mathrm{nA}$. We could speculate that, working to match the desired current setting, the feedback system may continuously push the applied voltage toward values producing the maximum possible number of charges in the electrospray gap, either by increasing the charging of existing ions through electrochemical processes [50], or by decreasing the size of electrosprayed droplets to facilitate the desolvation of charged species [7]. Further studies will be necessary to elucidate the process at the basis of these observations.

\section{Conclusions}

The preliminary results afforded by the feedback apparatus have provided validation to the current-sensing approach as a flexible way for dealing with challenging electrospray samples. Adjusting the emitter voltage as a function of real-time readings constitutes an efficient, rapid, and inexpensive way for maintaining acceptable signal stability, regardless of the actual morphology assumed by the spray plume. The comparison between TICs recorded with and without feedback activity has clearly illustrated the benefits of maximizing ion yield during the critical phase of ion accumulation in the external hexapole element of our FT-ICR mass spectrometer, which resulted in greater $\mathrm{S} / \mathrm{N}$ ratios and overall intensity. Data acquired in the presence of increasing electrolyte concentrations have demonstrated the ability of obtaining acceptable signal from samples containing up to $2.5 \mathrm{M}$ aqueous ammonium acetate. It is expected that the increased salt tolerance afforded by this approach will open the door for direct on-line application of separation techniques involving relatively high salt loads, such as ion-exchange chromatography, which thus far has been unpractical. Although the present design allowed for only a fixed target current to be set before analysis, it is possible to envision a program that would change such value according to a predefined 
function anticipating expected changes of solution properties, such as those occurring during gradient chromatography. In this direction, future studies will be necessary to systematically address the effects of salts and other additives on the current-voltage relationship under typical low-flow regimes. This information not only will guide the selection of initial settings for a certain experiment, but will also facilitate the implementation of ad hoc functions for varying the target current during gradient applications.

\section{Acknowledgments}

This research was funded by the National Science Foundation (Grant CHE-0439067).

\section{References}

1. Wilm, M. S.; Mann, M. Electrospray and Taylor-Cone Theory: Dole's Beam of Macromolecules at Last? Int. J. Mass Spectrom. Ion. Proc. 1994, 136, 167-180.

2. Emmett, M. R.; Caprioli, R. M. Micro-Electrospray Mass Spectrometry: Ultra-High-Sensitivity Analysis of Peptides and Proteins. J. Am. Soc. Mass Spectrom. 1994, 5, 605-613

3. Davis, M. T.; Stahl, D. C.; Hefta, S. A.; Lee, T. D. A Microscale Electrospray Interface for On-Line, Capillary Liquid Chromatography/ Tandem Mass Spectrometry of Complex Peptide Mixtures. Anal. Chem. $1995,67,4549-4556$.

4. Wilm, M.; Mann, M. Analytical Properties of the Nanoelectrospray Ion Source. Anal. Chem. 1996, 68, 1-8.

5. Wang, H.; Hackett, M. Ionization Within a Cylindrical Capacitor: Electrospray Without an Externally Applied High Voltage. Anal. Chem. 1998, 70, 205-212.

6. Fong, K. W.; Chan, T. W. A Novel Nonmetallized Tip for Electrospray Mass Spectrometry at Nanoliter Flow Rate. J. Am. Soc. Mass Spectrom. 1999, 10, 72-75.

7. De Juan, L.; Fernandez de la Mora, J. Charge and Size Distributions of Electrospray Drops. J. Colloid Interface Sci. 1997, 186, 280-293.

8. Yamashita, M.; Fenn, J. B. Electrospray Ion Source. Another Variation on the Free-Jet Theme. J. Phys. Chem. 1984, 88, 4671-4675.

9. Smith, R. D.; Loo, J. A.; Ogorzalek Loo, R. R.; Busman, M.; Udseth, H. R. Principles and Practice of Electrospray Ionization-mass Spectrometry for Large Polypeptides and Proteins. Mass Spectrom. Rev. 1991, 10, $359-452$.

10. Tang, L.; Kebarle, P. Effect of Conductivity of the Electrosprayed Solution on the Electrospray Current. Factors Determining Analyte Sensitivity in Electrospray Mass Spectrometry. Anal. Chem. 1991, 63, 2701-2715.

11. Tang, L.; Kebarle, P. Dependence of Ion Intensity in Electrospray Mass Spectrometry on the Concentration of the Analyte in the Electrosprayed Solution. Anal. Chem. 1993, 65, 3654-3668.

12. Grace, J. M.; Marijnissen, J. C. M. A Review of Liquid Atomization by Electrical Means. J. Aerosol Sci. 1994, 25, 1005-1019.

13. Cloupeau, M.; Prunet-Foch, B. Electrohydrodynamic Spraying Functioning Modes: A Critical Review. J. Aerosol Sci. 1994, 25, 1021-1036.

14. Fernandez de la Mora, J.; Loscertales, I. G. The Current Emitted by Highly Conducting Taylor Cones. J. Fluid Mech. 1994, 260, 155-184.

15. Noymer, P. D.; Garel, M. Stability and Atomization Characteristics of Electrohydrodynamic Jets in Cone-Jet and Multi-Jet Modes. J. Aerosol Sci. 2000, 31, 1165-1172.

16. Zeleny, J. Instability of Electrified Liquid Surfaces. Phys. Rev. 1917, 10, $1-6$.

17. Cloupeau, M.; Prunet-Foch, B. Electrostatic Spraying of Liquids in Cone-Jet Mode. J. Electrost. 1989, 22, 135-159.

18. Juraschek, R.; Röllgen, F. W. Pulsation Phenomena during Electrospray Ionization. Int. J. Mass Spectrom. 1998, 177, 1-15.

19. Jaworek, A.; Krupa, A. Classification of the Modes of EHD Spraying. J. Aerosol Sci. 1999, 30, 873-893.

20. Wei, J.; Shui, W.; Zhou, F.; Lu, Y.; Chen, K.; Xu, G.; Yang, P. Naturally and Externally Pulsed Electrospray. Mass Spectrom. Rev. 2002, 21, $148-162$.

21. Straub, R. F.; Voyksner, R. D. Negative Ion Formation in Electrospray Mass Spectrometry. J. Am. Soc. Mass Spectrom. 1993, 4, 578-587.

22. Parvin, L.; Galicia, M. C.; Gauntt, J. M.; Carney, L. M.; Nguyen, A. B.; Park, E.; Heffernan, L.; Vertes, A. Electrospray Diagnostics by Fourier
Analysis of Current Oscillations and Fast Imaging. Anal. Chem. 2005, 77, 3908-3915.

23. Alexander, M. S.; Paine, M. D.; Stark, J. P. Pulsation Modes and the Effect of Applied Voltage on Current and Flow Rate in Nanoelectrospray. Anal. Chem. 2006, 78, 2658-2664.

24. Nemes, P.; Marginean, I.; Vertes, A. Spraying Mode Effect on Droplet Formation and Ion Chemistry in Electrosprays. Anal. Chem. 2007, 79 3105-3116.

25. Marginean, I.; Nemes, P.; Vertes, A. Astable Regime in Electrosprays Phys. Rev. E Stat. Nonlin. Soft Matter Phys. 2007, 76, 026320.

26. Valaskovic, G. A.; Murphy, J. P. I.; Lee, M. S. Automated Orthogonal Control System for Electrospray Ionization. J. Am. Soc. Mass Spectrom. 2004, 15, 1202-1215.

27. Schrenk, W. Determining Voltage-Current Characteristics of an Electrospray Source. European Patent EP 1173111 A1. Agilent Technologies, Inc.: Waldbronn, Germany, 2006.

28. Marginean, I.; Kelly, R. T.; Page, J. S.; Tang, K.; Smith, R. D. Electrospray Characteristic Curves: In Pursuit of Improved Performance in the Nanoflow Regime. Anal. Chem. 2007, 79, 8030-8036.

29. Gapeev, A.; Berton, A.; Fabris, D. Current-Controlled Nanospray for the Analysis of Less Than Ideal Samples. Proceedings of the 56th American Society for Mass Spectrometry Conference, Denver, CO, June 2007; ASMS: Sante Fe, NM, 2008; pp 1-5.

30. Staats, S. L. T.; Fogiel, A. J. Apparatus and Method for Controlling and Electrostatically Induced Liquid Spray. U.S. Patent Application 20080203198. Phoenix S\&T, Inc., Elkton, MD, 2008.

31. Jackson, G. S.; Enke, C. G. Electrical Equivalence of Electrospray Ionization with Conducting and Nonconducting Needles. Anal. Chem. 1999, 71, 3777-3784.

32. Liptak, B. C.; Venczel, K. Instrument Engineers Handbook; Chilton Book Company, Radnor, PA, 1985; pp 14-20.

33. Comisarow, M. B.; Marshall, A. G. Fourier Transform Ion Cyclotron Resonance. Chem. Phys. Lett. 1974, 25, 282-283.

34. Senko, M. W.; Hendrickson, C. L.; Emmett, M. R.; Stone, D.-H. Marshall, A. G. External Accumulation of Ions for Enhanced Electrospray Ionization Fourier Transform Ion Cyclotron Resonance Mass Spectrometry. J. Am. Soc. Mass Spectrom. 1997, 8, 970-978.

35. Sannes-Lowery, K.; Griffey, R. H.; Kruppa, G. H.; Speir, J. P.; Hofstadler S. A. Multipole Storage Assisted Dissociation, a Novel In-Source Dissociation Technique for Electrospray Ionization Generated Ions. Rapid Commun. Mass Spectrom. 1998, 12, 1957-1961.

36. Smith, D. P. H. The Electrohydrodynamic Atomization of Liquids. IEEE Trans. Ind. Appl. 1986, 22, 527-535.

37. Fernandez de la Mora, J. The Fluid Dynamics of Taylor Cones. Annu. Rev. Fluid Mech. 2007, 39, 217-243.

38. Stults, J. T.; Marsters, J. C. Improved Electrospray Ionization of Synthetic Oligodeoxynucleotides. Rapid Commun. Mass Spectrom. 1991, 5 , 359-363.

39. Nordhoff, E.; Kirpekar, F.; Roepstorff, P. Mass Spectrometry of Nucleic Acids. Mass Spectrom. Rev. 1996, 15, 67-138.

40. Turner, K. B.; Monti, S.; Fabris, D. Like Polarity Ion/Ion Reactions Enable the Investigation of Specific Metal Interactions in Nucleic Acids and Their Non-covalent Assemblies. J. Am. Chem. Soc. 2008, 30, 1335313363.

41. English, W. N. Corona from a Water Drop. Phys. Rev. 1948, 74, 179-189.

42. Ikonomou, M. G.; Blades, A. T.; Kebarle, P. Electrospray-Ion Spray: A Comparison of Mechanisms and Performance. Anal. Chem. 1991, 63, 1989-1998.

43. Meesters, G. M. H.; Vercoulen, P. H. W.; Marijnissen, J. C. M.; Scarlett, B. A (Sub-)Micron Aerosol Generator, Using a High Electric Field. J. Aerosol Sci. 1991, 22, S11-S14.

44. Meesters, G. M. H.; Vercoulen, P. H. W.; Marijnissen, J. C. M.; Scarlett, B. Generation of Micron-Sized Droplets from the Taylor Cone. J. Aerosol Sci. 1992, 23, 37-49.

45. Kriger, M. S.; Cook, K. D.; Ramsey, R. S. Durable Gold-Coated Fused Silica Capillaries for Use in Electrospray Mass Spectrometry. Anal. Chem. 1995, 67, 385-389.

46. Chen, D.-R.; Pui, D. Y. H.; Kaufman, S. L. Electrospraying of Conducting Liquids for Monodisperse Aerosol Generation in the $4 \mathrm{~nm}$ to $1.8 \mu \mathrm{m}$ Diameter Range. J. Aerosol Sci. 1995, 26, 963-977.

47. Constantopoulos, T. L.; Jackson, G. S.; Enke, C. G. Effects of Salt Concentration on Analyte Response Using Electrospray Ionization Mass Spectrometry. J. Am. Soc. Mass Spectrom. 1999, 10, 625-634.

48. Lopez-Herrera, J. M.; Barrero, A.; Boucard, A.; Loscertales, I. G.; Marquez, M. An Experimental Study of the Electrospraying of Water in Air at Atmospheric Pressure. J Am. Soc. Mass Spectrom. 2004, 15, 253-259.

49. Paine, M. D.; Alexander, M. S.; Stark, J. P. Nozzle and Liquid Effects on the Spray Modes in Nanoelectrospray. J. Colloid Interface Sci. 2007, 305, 111-123.

50. Van Berkel, G. J.; Giles, G. E.; Bullock, J. S., IV; Gray, L. J. Computational Simulation of Redox Reactions within a Metal Electrospray Emitter. Anal. Chem. 1999, 71, 5288-5296. 